\title{
Local probability effects of repeating irrelevant attributes
}

\author{
Wolf Schwarz ${ }^{1}$ Dennis Reike ${ }^{1}$
}

Published online: 13 October 2016

(C) The Psychonomic Society, Inc. 2016

\begin{abstract}
We present two experiments in which participants classify stimuli having two potentially conflicting attributes, one of which is response-relevant whereas the other ("irrelevant") attribute is logically and statistically independent of the response. We introduce a novel design not used with filtering tasks before in which the main factor is the local (i.e., one-step) transition probability $\pi(=0.25,0.50,0.75)$ that the irrelevant attribute is repeated from one trial to the next. Experiment 1 involved a visual Simon task in which the color of the stimulus is relevant and its location is irrelevant. Experiment 2 used a semantic classification task in which the parity of a digit presented is relevant and its numerical magnitude is irrelevant. The results of both experiments demonstrate that participants in the $\pi=0.75$ group responded faster when the irrelevant attribute is in fact repeated rather than alternated; in contrast, participants in the $\pi=0.25$ group responded faster (Experiment 1) or equally fast (Experiment 2) when the irrelevant attribute is alternated rather than repeated. These expectancy-related effects cannot be attributed to spurious design contingencies as the irrelevant attribute was independent of the relevant attribute (and thus of the response), of the congruency status, and also of their alternation/repetition. One interpretation of our findings is that information about the irrelevant attribute in the previous trial is used much as an informative
\end{abstract}

Wolf Schwarz

wschwarz@uni-potsdam.de

Dennis Reike

reike@uni-potsdam.de

1 Department of Psychology, University of Potsdam, PO Box 6015 53, 14415, Potsdam, Germany central precue, so that participants can prepare early processing stages in the current trial, with the corresponding benefits and costs typical of standard cueing studies.

Keywords Repetition effect - Local stimulus probability · Expectancy $\cdot$ Filtering tasks $\cdot$ Simon effect $\cdot$ SNARC effect

Among the most widely used experimental designs in human information processing are filtering tasks in which the stimuli presented to the observers for classification vary on two dimensions, or attributes, one of which is responserelevant, and one is not (e.g., Kahneman \& Treisman, 1984; Pashler, 1998). Often, these attributes are independent, both logically and statistically, but are related by other, potentially conflicting aspects, in which case the generic term "conflict task" is sometimes used (e.g., Jiménez \& Méndez, 2013; Miller, 1991; Schwarz \& Ischebeck, 2003). These aspects may be defined by the relation of the irrelevant attribute to the relevant attribute (e.g., Stroop, 1935; Eriksen \& Eriksen, 1974) or to the responses (e.g., spatial correspondence; Simon \& Berbaum, 1990). Thus, standard designs such as the Stroop, the Simon, or the flanker task are all specific cases of conflict tasks, although this formal analogy should not conceal the several systematic differences that exist between them (for detailed review, see, e.g., Kornblum \& Stevens, 2002; Pratte, Rouder, Morey, \& Feng, 2010). In many conditions typical of conflict tasks, the irrelevant stimulus attribute, even if uninformative about the relevant attribute and the response, nevertheless systematically influences the participant's performance, such that congruent irrelevant attributes lead to faster and more accurate responses than incongruent ones. Thus, speaking of attributes as "irrelevant" does not imply that they do not influence the way in which a stimulus is processed-it 
merely indicates that from knowledge about these attributes alone the correct response cannot be deduced at a better than chance level.

\section{Effects of repeating irrelevant attributes}

Early accounts of filtering and conflict tasks tended to focus on factors related to the specific conditions prevailing within any given trial (for reviews, see, e.g., Lu \& Proctor, 1995; MacLeod, 1991; Pashler, 1998). However, subsequent research has documented systematic sequential effects in many of these tasks. Extensive literature addresses sequential effects in conflict paradigms defined by the congruency status (i.e., congruent vs. incongruent) of the previous trial; a basic finding is that congruency effects tend to be reduced following incongruent as compared to congruent trials (e.g., Botvinick, Braver, Barch, Carter, \& Cohen, 2001; Duthoo, Abrahamse, Braem, Boehler, \& Notebaert, 2014; Gratton, Coles, \& Donchin, 1992; Jiménez \& Méndez, 2013; Mayr, Awh, \& Laurey, 2003; Pfister, Schröder, \& Kunde, 2013; Puccioni \& Vallesi, 2012).

In contrast, the present study addresses effects related to whether the irrelevant attribute of the previous trial is repeated or alternated. Extant findings regarding these repetition effects present a mixed picture, with several differences between paradigms and conditions. For example, using a standard Simon task Notebaert, Soetens, and Melis (2001; for related studies and reviews, see, e.g., Hommel, 2011; Notebaert \& Soetens, 2003; Wühr \& Ansorge, 2005; Zeischka, Deroost, Maetens, \& Soetens, 2010) studied the effect of repeating the color (relevant attribute) and/or the location (irrelevant attribute) of the visual stimulus. They found (Experiments 1 and 2) that repeating the location had no main effect on RT but interacted with repeating the relevant attribute (and thus the response) such that RT was shorter when both attributes were repeated or both alternated (for similar findings, see Kleinsorge, 1999; Schwarz \& Ischebeck, 2000). Furthermore, for response-to-signal intervals (RSIs) typical of standard conflict paradigms (1000 ms) repeating vs. alternating the irrelevant location did not influence the size of the Simon effect, whereas for very short RSIs (50 ms) a Simon effect was observed for location alternations but not for repetitions.

Related results on repeating irrelevant attributes were obtained by Kornblum and Stevens (2002, Experiment 4) in a four-choice task in which central letters (relevant attribute) were flanked either by response-incongruent arrows, or by incongruent letters that were targets on other trials. Repeating irrelevant arrow flankers had neither a main nor any interaction effect. However, repeating irrelevant flanking letters reduced RT by $26 \mathrm{~ms}$ relative to alternating them, but even then there was no interaction between repeating the relevant and repeating the irrelevant attribute, in contrast to the study of Notebaert et al. (2001) using the Simon task.

Finally, effects of repeating irrelevant attributes have also been observed in non-spatial filtering tasks (Frings, Rothermund, \& Wentura, 2007; Rothermund, Wentura, \& De Houwer, 2005), for example, in semantic classification, such as numerical comparison (Dehaene, 1996; Pashler \& Baylis, 1991; Pfister et al., 2013; Schwarz \& Ischebeck, 2000; Tan \& Dixon, 2011). For example, in Schwarz and Ischebeck (2000), participants classified numbers (presented as digits or number words; the irrelevant attribute) as smaller or larger than 5 (relevant attribute). Although uninformative about the response, repeating the logically irrelevant notation (arabic vs. verbal) produced a partial repetition benefit, even when the number itself was not repeated (for related partial repetition effects in digit naming, see Marcel \& Forrin, 1974; Meuter \& Allport, 1999).

\section{Local repetition probability}

The present study further investigates these sequential effects of repeating irrelevant stimulus attributes reviewed above by a technique that has not previously been used in the context of filtering and conflict tasks, namely by systematically varying the local, i.e., trial-to-trial repetition probability of the response-irrelevant attribute. In a classical paper, Bertelson (1961) first showed that local stimulus transition probabilities may be varied without altering the global (i.e., overall) probabilities of the stimulus alternatives involved. Specifically, he considered two-choice designs in which according to a first-order two-state Markov chain in each trial the local probability of repeating the previous stimulus was equal to $0<\pi<1$ while the global probability of each stimulus was held constant at 0.50 . As seems natural, Bertelson (1961) used stimuli that varied only in one (thus, response-relevant) attribute, namely, the location at which they were presented. For RSIs typical of conflict tasks (500 ms), he found (his Table 4, group TL) that relative to a control group ( $\pi=0.50$ ) observers in a $\pi=0.75$ group responded faster to stimulus repetitions and slower to stimulus alternations, whereas an exactly complementary pattern was observed in a $\pi=0.25$ group. Bertelson (1961) concluded that with sufficiently long RSIs his observers selectively prepared for the locally more likely stimulus alternative, even when for all three groups both stimuli were presented equally often. Subsequent research has confirmed and extended these basic findings, for example, to designs with more than two stimuli (Kornblum, 1968), to sequential trial dependencies of higher order (e.g., Jentzsch \& Leuthold, 2005), and to analyses of event-related brain 
potentials (e.g., Leuthold \& Sommer, 1993); for detailed reviews, see, for example, Jiménez and Méndez (2013), Jones, Curran, Mozer, \& Wilder (2015), or Soetens (1998). Notwithstanding these confirmations and extensions, a central aspect of these studies in the present context remains that all of them focused on the local repetition probability of the attribute that was actually response-relevant.

The main question, then, addressed by the present experiments is: Do Bertelson's (1961) conclusions regarding the local repetition probability of response-relevant attributes extend to response-irrelevant attributes as well? Specifically, adaptations to local repetition probabilities are often thought to reflect expectancies (for recent reviews, see Jiménez \& Méndez, 2013; Jones et al., 2015) enabling the participants to selectively prepare for more likely decisions or responses. Given that by definition irrelevant attributes per se provide no basis to anticipate the forthcoming response, one plausible view is that variations of the local repetition probability of irrelevant attributes will have no additional differential effect, over and above the welldocumented basic effects of repeating irrelevant attributes reviewed above, all of which were obtained for $\pi=0.50$. According to this view, repeating, for example, the irrelevant location in a Simon task would still show the effects (e.g., Notebaert et al., 2001) reviewed above, but these effects would not in turn be further modified by varying the local probability $\pi$ of repeating the irrelevant location. Consistent with this view, note that repeating the relevant attribute necessarily implies repeating the response as well; in contrast, in the present experiments repetitions of the irrelevant attribute are unrelated to the response. Therefore, to the degree that the selective preparatory effects of local probability first observed for relevant attributes by Bertelson (1961) mainly reflect response-related expectancies it is quite conceivable that these effects will not extend to variations of the local repetition probability $\pi$ of attributes which are response-irrelevant.

On the other hand, at least some evidence suggests that variations of the local repetition probability of responserelevant attributes might have some effect. First, an extensive literature, referred to above, has clearly established that repeating irrelevant attributes per se does have reliable and systematic effects, under a wide range of well-understood conditions. Therefore, selectively adapting to the local probability of such repetitions might potentially enhance the efficiency of those processing mechanisms underlying such effects. For example, in the context of a standard Simon task, varying the local repetition probability of the irrelevant attribute does provide probabilistic information about the stimulus location in the next trial, not unlike in principle to informative central location precues (see, e.g., Jongen \& Smulders, 2007; Lu \& Proctor, 1995).
Second, recent research on the sequential congruency effect (for recent review, see Duthoo et al., 2014; Hommel, 2011; Pfister et al., 2013; Puccioni \& Vallesi, 2012) supports the view that the local repetition probability of higherorder attributes which are unrelated to the response per se can have systematic effects, too. For example, Jiménez and Méndez (2013) studied the sequential congruency effect in a Stroop task by varying the local repetition probability of a trial to be congruent or incongruent, without altering the overall probability $(0.50)$ of congruent vs. incongruent trials (for a related study using explicit cues indicating the congruency status of the next trial, see Wühr \& Kunde, 2008). In their Experiment 2A the congruency status (i.e., congruent vs. incongruent) of the previous trial was repeated with a local probability of $\pi=0.70$. Compared to a neutral $\pi=0.50$ condition (their Experiment 1), this led to an increase of the congruency effect following congruent trials, and to a decrease of the congruency effect following incongruent trials. Jiménez and Méndez (2013) account for this effect by assuming that participants form expectancies on the basis of learned design contingencies. Thus, following a congruent $n-1$ trial, participants tend to expect another congruent trial which further decreases their congruent trial $n$ RT and increases their incongruent trial $n$ RT, producing an overall increase of the congruency effect in trial $n$. In contrast, following an incongruent $n-1$ trial, participants tend to expect another incongruent trial which decreases their incongruent trial $n$ RT and increases their congruent trial $n$ RT, thus producing a decrease of the congruency effect in trial $n$. As expected, complementary results were observed for a $\pi=0.30$ condition (Experiment $2 \mathrm{~B}$ ) which led to a reduction (an increase) of the congruency effect following a congruent (an incongruent) $n-1$ trial.

\section{The present experiments}

The detailed findings just reviewed directly relate to the issues addressed in the present study, and several critical features should be noted in relation to the experiments reported below. First, the RSI of $0 \mathrm{~ms}$, chosen by Jiménez and Méndez (2013) to minimize the effect of expectancies, is untypical of standard conflict tasks; at present it is an open question whether local repetition probability influences RT at larger RSIs (but see Jiménez \& Méndez, 2014). Furthermore, note that the specific property whose local repetition probability was varied in the study of Jiménez and Méndez (2013) was not the irrelevant attribute (i.e., the word meaning) as such but rather the congruency status of the trial. To study the effect (if any) of the local repetition probability of response-irrelevant attributes a filtering task design is required in which the irrelevant attribute is locally 
(from one trial to the next) repeated with a given probability $\pi$, independent of the response-relevant attribute, and also independent of the congruency status of the trial. In the absence of extant studies with these design features, it is an open question whether variations of the local repetition probability of response-irrelevant attributes have any specific differential effects beyond repetition per se at all, and if so whether these effects are similar to those for responserelevant attributes. The present experiments sought to fill in this gap, using two representative and well-investigated paradigms.

In our Experiment 1 we used a standard Simon task, in which the relevant attribute is color, and the irrelevant attribute is location. Our Experiment 2 addressed the same issues in the context of semantic classification, and exploited the well-documented SNARC effect (spatialnumerical association of response codes; Dehaene, Bossini, \& Giraux, 1993; Dehaene, 2011; Fias \& Fischer, 2005) in which participants indicate the parity (relevant attribute) of single digits by manual responses (for saccadic and pedal responses, see Schwarz \& Keus, 2004; Schwarz \& Müller, 2006), the irrelevant attribute in this case is whether the digit is numerically small $(<5)$ or large $(>5)$.

For an unambiguous attribution of the effects of varying the local probability of the response-irrelevant attribute it is of central importance to use stimulus sequences which meet several crucial boundary conditions (for a set of systematic studies of contingency learning effects in this context, see Schmidt, 2013; Schmidt \& Besner, 2008; Schmidt, Crump, Cheesman, \& Besner, 2007; Schmidt \& De Houwer, 2012a, b; Schmidt, De Houwer, \& Besner, 2010). In particular, we generated stimulus sequences in which overall both levels of the relevant attribute (and thus both responses) were presented equally often, the same was true of the levels of the irrelevant attribute, and also for congruent vs. incongruent trials. Furthermore, in our design repeating vs. alternating the irrelevant attribute was independent of repeating vs. alternating the relevant attribute (and thus the response), and also independent of whether the trial was congruent or not. Finally, in all conditions, the relevant attribute was repeated with a probability of 0.50 ; the same was true of the congruency status (e.g., Jiménez \& Méndez, 2013).

\section{Experiment 1}

Experiment 1 was a visual Simon task, with color as the relevant and location as the irrelevant attribute. The factor of central importance, varied between participants, was the probability $\pi(=0.25,0.50,0.75)$ that the irrelevant attribute (i.e., location) was repeated from one trial to the next.

\section{Method}

\section{Participants}

Thirty-six University of Potsdam students, aged 18-33, with normal or corrected-to-normal vision participated in one session of approximately one hour. They either received a payment of 8 Euro or course credit for their participation.

\section{Stimuli and apparatus}

The stimuli were circular color (red/green) patches (1.2 deg diameter) presented against a gray background $4 \mathrm{deg}$ to the right or left of a centered fixation cross on a $75 \mathrm{~Hz}$, $1028 \times 768$ pixel VGA monitor. Participants responded by pressing buttons on custom-built response boxes with the index fingers of their left or right hand; the response boxes were fixed on a table $25 \mathrm{~cm}$ to the left and right of the midline.

\section{Procedure}

Twelve participants were randomly assigned to each group defined by the probability $\pi(=0.25,0.50,0.75)$ that the location was repeated from one trial to the next. Following one practice block, in each of ten blocks (comprising three warm-up trials, followed by 72 regular trials) each of the four combinations of color and location was shown 18 times, so that each color and each location occurred 36 times. Color (and thus the response) was repeated/alternated 36 times, the same was true of congruency status. For the $\pi=0.25(0.50,0.75)$ group, location was repeated in 18 $(36,54)$ of the 72 trials in each block. Sequences were generated such that location repetitions were independent of both color and color repetitions, and also of congruency status and congruency status repetitions.

The task was to indicate, as quickly as possible, the color of the stimulus by a button press. Six participants in each group worked under each of both color-to-response mappings (this variable had no main or interaction effect, and is ignored in the following). Each trial started with the presentation of a central fixation cross. After a delay of $500 \mathrm{~ms}$ the stimulus was presented until a button was pressed; the next trial then started $1000 \mathrm{~ms}$ after the response.

All effects were tested at a significance level of $\alpha<.05$. For a trial to enter into the analysis of mean RT the response had to be correct, and be preceded by a correct response (exclusion rate of 8.4 ); also, RTs outside the range of 150 $\mathrm{ms}$ to $900 \mathrm{~ms}$ were excluded (a rate of $0.9 \%$ ); alternative exclusion rules produced the same pattern of significant results. 


\section{Results}

\section{Mean RT}

We analyzed mean RT by a $3 \times 2 \times 2 \times 2$ mixed-model ANOVA in which the repetition probability of the irrelevant location was a between-subjects factor $(3: 0.25,0.50,0.75)$; three binary within-subject factors were defined by whether or not in the trial considered the irrelevant attribute (location) was repeated (2), the relevant attribute (color, and thus the response) was repeated (2), and whether the trial was congruent or incongruent (2).

Overall, response (i.e., color) repetitions (379 ms) were faster than alternations $(402 \mathrm{~ms} ; \mathrm{F}(1,33)=37.90, \mathrm{MSE}=$ $1045, \eta^{2}=0.54, \mathrm{p}<.001$; Fig. 1, right panel), and congruent trials (382 ms) led to shorter RTs than incongruent trials $(399 \mathrm{~ms}$, Simon effect; $\mathrm{F}(1,33)=40.11, \mathrm{MSE}=541$, $\eta^{2}=0.55, \mathrm{p}<.001 ;$ Fig. 2$)$. In contrast, repeating (390 $\mathrm{ms})$ vs. alternating $(391 \mathrm{~ms})$ the irrelevant location had no main effect on $\mathrm{RT}\left(\mathrm{F}(1,33)=.28, \mathrm{MSE}=519, \eta^{2}=0.01\right.$, $\mathrm{p}<.598$ ), confirming the previous findings reviewed in the Introduction.

The most relevant finding in the present context is the interaction of the local repetition probability $\pi$ for the irrelevant location with its actual repetition or alternation in a given trial $\left(\mathrm{F}(2,33)=6.94, \mathrm{MSE}=519, \eta^{2}=0.30\right.$, $\mathrm{p}<.003$ ), shown in the left panel of Fig. 1. For the $\pi=0.25$ group repeating the location increased mean RT

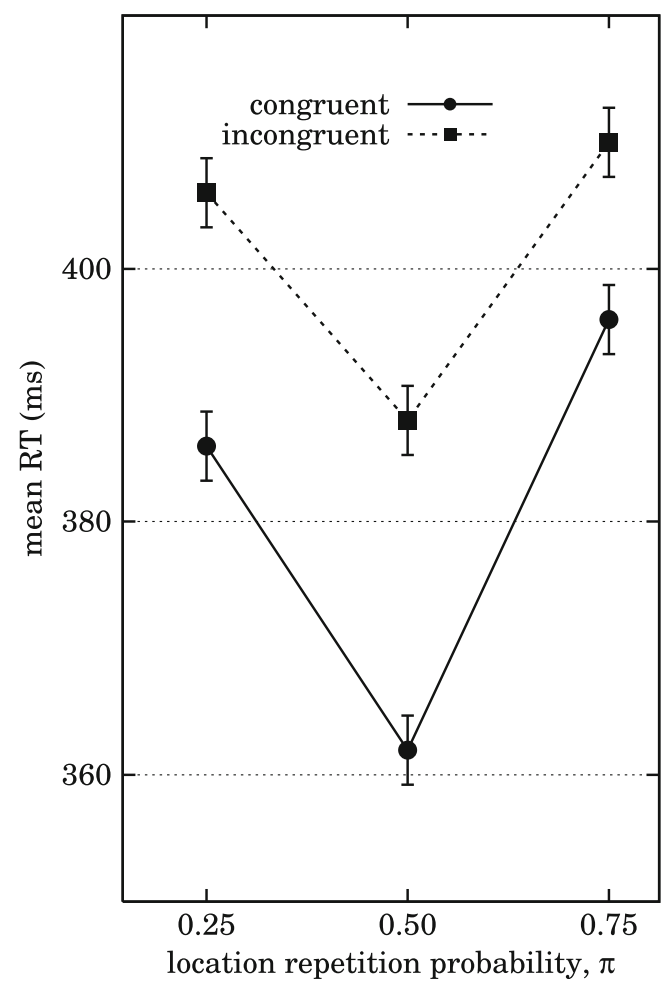

Fig. 2 Mean RT (ordinate in ms) for three groups with local probability of location repetition equal to $\pi=0.25,0.50,0.75$ (abscissa). Dots and solid lines indicate congruent, squares and dotted lines incongruent trials. Error bars show \pm 1 s.e.m. (Loftus \& Massey,1994)

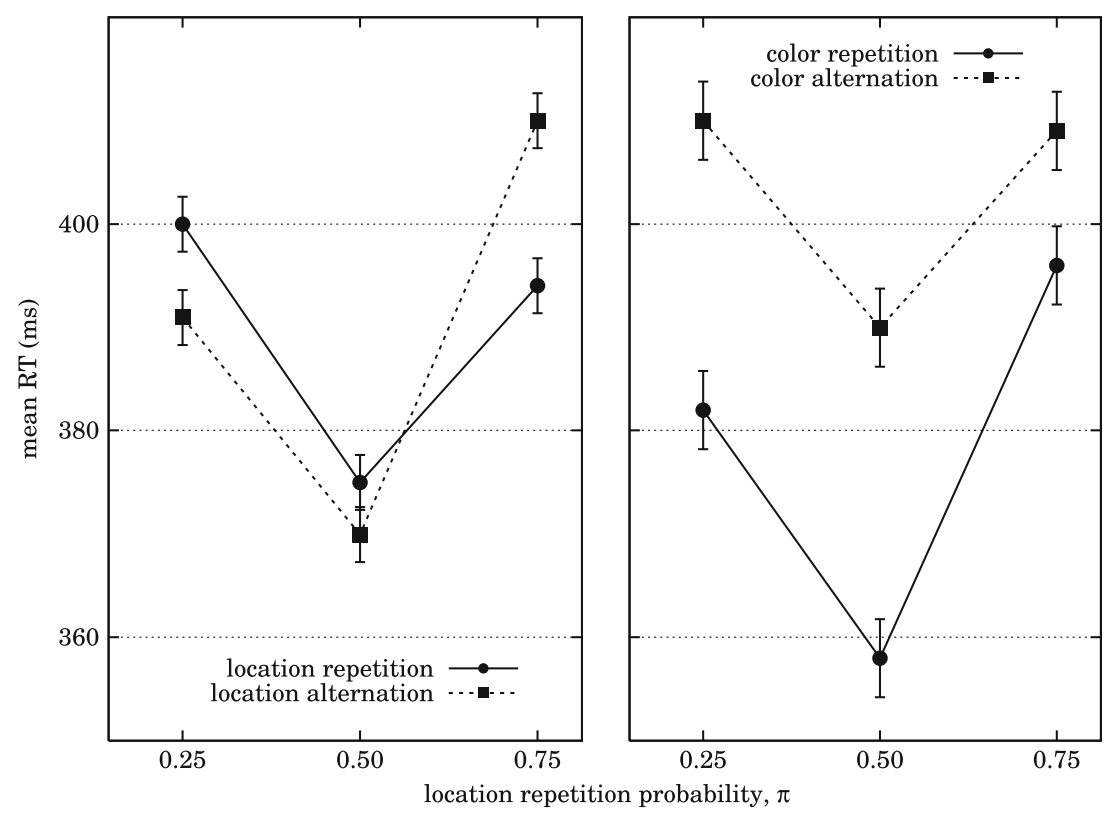

Fig. 1 Both panels: mean RT (ordinate in ms) for three groups with local probability of location repetition equal to $\pi=0.25,0.50,0.75$ ( $a b s c i s s a$ ). Left: mean RT for location repetition (dots, solid lines) vs. location alternation (squares, dotted lines) trials. Right: mean RT for color (thus, response) repetition (dots, solid lines) vs. color alternation (squares, dotted lines) trials. Error bars show \pm 1 s.e.m. (Loftus \& Massey, 1994) 
by $8 \mathrm{~ms}$, relative to alternating the location. In contrast, for the $\pi=0.75$ group repeating the location decreased mean RT by $15 \mathrm{~ms}$. For the $\pi=0.50$ group repeating $(375 \mathrm{~ms})$ or alternating $(372 \mathrm{~ms})$ the location had no reliable effect $\left(t(11)=1.09, \eta^{2}=0.10, \mathrm{p}=.30\right)$. The group factor defined by the three levels of $\pi$ neither modified the response repetition main effect (Fig. 1, right panel; $\mathrm{F}(2,33)=2.43$, MSE $\left.=1045, \eta^{2}=0.13, \mathrm{p}<.104\right)$ nor the Simon main effect (Fig. 2; F(2,33) = 0.66, $\mathrm{MSE}=541, \eta^{2}=0.04, \mathrm{p}<.523$ ).

Only two further interaction effects were significant, both confirming effects reviewed in the Introduction, and none of which involved the group factor for the levels of $\pi$. First, the congruency effect is larger $(22 \mathrm{~ms})$ when the location is alternated compared to when it is repeated $(13 \mathrm{~ms} ; \mathrm{F}(1,33)$ $\left.=8.92, \mathrm{MSE}=142, \eta^{2}=0.21, \mathrm{p}<.005\right)$. Second, when the location is repeated, color (and thus response) repetitions are $55 \mathrm{~ms}$ faster; this turns into an advantage for color (and response) alternations of $8 \mathrm{~ms}$ when the location alternates $\left(\mathrm{F}(1,33)=279.73, \mathrm{MSE}=244, \eta^{2}=0.89\right.$, $\mathrm{p}<.001)$. In contrast, we found no significant three-way interaction of the location repetition probability $\pi$ with the location's actual repetition or alternation in a given trial, and congruency $\left(\mathrm{F}(2,33)=0.11, \mathrm{MSE}=142, \eta^{2}=0.00\right.$, $\mathrm{p}=.90)$.

\section{Error rates}

The overall error rate (in \%) was 4.3. The results of the ANOVA of error rates mostly conform to those for RT, such that long RTs go with high error rates. Specifically, fewer errors were made in congruent (3.7) than in incongruent (5.0) trials $\left(\mathrm{F}(1,33)=4.78, \mathrm{MSE}=21.50, \eta^{2}=0.13\right.$, $\mathrm{p}<.036)$. Of central importance is the interaction of the irrelevant repetition probability $\pi$ with repeating vs. alternating the irrelevant location $(\mathrm{F}(2,33)=8.98, \mathrm{MSE}=7.26$, $\left.\eta^{2}=0.35, \mathrm{p}<.001\right)$. This interaction reflects that participants in the $\pi=0.25$ group produced more errors when the irrelevant attribute (location) was repeated (5.4) than when it alternated (4.2), whereas for the $\pi=0.75$ group this pattern was exactly reversed (3.7 for repeated vs. 5.8 for alternated locations). As was the case for RT, the $\pi=0.50$ group showed no reliable error rate effect for repeating (3.3) vs. alternating (3.8) the location.

Only two further interaction effects were significant, none involving the group factor. First, when the color is repeated then incongruent trials lead to more errors (5.7) than congruent ones (3.4). However, when the color alternates, the error rates in incongruent (4.3) and congruent trials (4.1) are nearly equal, resulting in an interaction of relevant (i.e., color) repetition with congruency $(\mathrm{F}(1,33)=$ 10.67, $\left.\mathrm{MSE}=7.52, \eta^{2}=0.24, \mathrm{p}<.003\right)$. Second, confirming the analogous effect for mean RTs, when the location is repeated, fewer errors (2.2) are made when color is repeated, too, than when color alternates (6.0). Conversely, when the location alternates, more errors are made for color repetitions (6.8) than for color alternations (2.4), producing a highly reliable interaction $(\mathrm{F}(1,33)=42.91, \mathrm{MSE}=28.55$, $\left.\eta^{2}=0.57, \mathrm{p}<.001\right)$.

\section{Discussion}

The results of Experiment 1 provide a first demonstration that variations of the local transition probability $\pi$ of repeating response-irrelevant attributes (location) can have reliable and consistent effects on performance in a standard filtering task. That is, the present results indicate that location is not irrelevant in the sense that expectations about the location of the forthcoming stimulus based on the firstorder transition probabilities have no systematic effects on performance (for a discussion of the conditions under which these expectations become explicit, see, e.g., Blais, Harris, Guerrero, \& Bunge, 2012; Bugg, Diede, Cohen-Shikora, \& Selmeczy, 2015; Schmidt et al., 2007). Specifically, participants in the group with a high local probability of repeating the irrelevant location responded faster and more accurately in trials in which the location is in fact repeated, and a converse pattern was observed in the group for which $\pi$ was low.

Given that this effect in turn neither modified the response repetition benefit nor the congruency effect, one plausible interpretation, then, is that the local repetition probability for location speeds up an early orienting stage, much like informative central location cues do (e.g., Girardi, Antonucci, \& Nico, 2013, 2015). More specifically, the participants in groups $\pi=0.25$ and 0.75 might interpret the location of the current stimulus as a (mostly) valid cue for the stimulus location in the next trial, and benefit from a corresponding attentional orientation whenever the stimulus in fact occurs at the more likely location. Reviewing precueing effects in the Simon task, Lu and Proctor (1995) concluded that "precues influence the magnitude of the Simon effect only when an expectancy for a particular response is created ... but not by an expectancy for the particular location in which the target stimulus will occur" (p. 187, italics added). Similarly, Wühr and Kunde (2008) observed that in a Simon task participants cannot make use of reliable cues about the congruency of a forthcoming stimulus to change the attentional weights of processing channels for different stimulus dimensions, which is in line with Jiménez and Méndez (2013, p. 281) who conclude that although "the manipulated contingencies had been effective in changing participants' expectancies concerning the congruency of the next trial, the effects of congruency remained relatively immune to such contingencies". Thus, our own findings and the interpretation given fit in well with related standard findings from the Simon task that valid central location precuing leads to an 
overall speed benefit, but does not modify the size of the Simon effect.

To assess the generality of our findings, it would be important to extend the basic design used in Experiment 1 in several ways. First, given the findings summarized in the Introduction regarding partial repetition benefits in semantic classification, it seems natural to ask if the effects of the local repetition probability for irrelevant attributes extend beyond simple sensorimotor (e.g., Simon) tasks. More specifically, while the effects observed in Experiment 1 could possibly reflect shifts of spatial attention to the location predicted by the preceding trial, analogous effects with non-spatial predictability would presumably require a more general explanation.

Second, note that the standard Simon task used in Experiment 1 involves only four different stimuli which means that repeating both the relevant and the irrelevant attribute implies an exact replica of the previous trial, which potentially provides a repetition benefit specific to identical repetitions (see, e.g., Hommel, 2011; Pashler \& Baylis, 1991; Schwarz \& Ischebeck, 2000).

It is, therefore, conceivable that our findings regarding the critical interaction of the local repetition probability $\pi$ for the irrelevant location with its actual repetition or alternation in a given trial (left panel of Fig. 1) reflects an effect that is closely tied to identical repetitions. ${ }^{1}$ To test this conjecture, we run an additional analysis in which all trials were removed in which the stimulus presented was identical (i.e., in location and color) to the stimulus in the preceding trial. In the design of Experiment 1 repeating the relevant attribute color and repeating the irrelevant attribute location were always independent events: in all three $\pi=0.25 / 0.50 / 0.75$ groups, there was an equal proportion (i.e., 0.50) of relevant repetitions and relevant alternations both within irrelevant repetitions and within irrelevant alternations. Therefore, the exclusion of identical repetitions maintained the same trial composition between the three $\pi$-groups. Note that excluding identical repetitions does not affect at all the RT means for alternations of the irrelevant attribute, which by definition cannot be identical repetitions. Rather, it only excludes half of all those trials in which the irrelevant attribute is repeated, namely, that half in which the relevant attribute (and thus the response) is also repeated, leaving for irrelevant repetitions only the other half in which the relevant attribute (and thus the response) alternates. As described above, there is a robust interaction of repeating the relevant and the irrelevant attribute such that repeating both, or alternating both, leads to clearly faster RTs than in the two

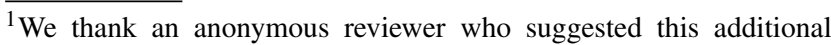
analysis.
}

mixed cases. As a result, whereas mean RTs for alternations of the irrelevant attribute remain by definition unchanged by excluding identical repetitions, mean RTs for repetitions of the irrelevant attribute are clearly longer (about 30 ms) after the exclusion of identical repetitions. The crucial finding, however, is that this effect is essentially the same across all three $\pi$-groups, thus producing very nearly the same interaction contrast $(\mathrm{F}(2,33)=11.51, \mathrm{MSE}=165$, $\left.\eta^{2}=0.41, \quad \mathrm{p}<.0002\right)$ of the local repetition probability $\pi$ for the irrelevant location with its actual repetition or alternation in a given trial as in the original analysis including identical repetitions. Specifically, after excluding identical repetitions the RT advantage of alternations over repetitions of the irrelevant location was maximal ( $38 \mathrm{~ms}$ ) for $\pi=0.25$ (where such alternations are expected), decreased to $33 \mathrm{~ms}$ for $\pi=0.50$, and was minimal $(5 \mathrm{~ms})$ for $\pi=0.75$. This analysis strongly suggests that the interaction of $\pi$ with the actual location repetition or alternation in a given trial found in Experiment 1 cannot be attributed to a processing benefit specific to identical repetitions. Clearly, this conclusion could be further strengthened by using a richer design involving a categorically defined irrelevant attribute such that this attribute can be repeated with non-identical stimuli, even when the relevant attribute (i.e., the response) is repeated, too.

\section{Experiment 2}

In Experiment 2 participants classified digits (1-9, excluding 5) according to their parity (relevant dimension), indicating their decision by manual responses (index finger of the left or right hand). Under standard conditions the difference of right-hand RT minus left-hand RT decreases with the numerical magnitude of the digit (the so-called SNARC effect; Dehaene et al., 1993; for review, see Dehaene, 2011; Fias \& Fischer, 2005; Schwarz \& Keus, 2004). That is, ignoring potential main effects of magnitude and hand, participants respond (relatively) faster to small digits $(<5)$ with their left hand, and to large digits $(>5)$ with their right hand, even though numerical magnitude per se is a responseirrelevant attribute. A common interpretation is that the size of the SNARC effect reflects the correspondence (or lack thereof) of the internal representation of numerical magnitude in the format of a left-to-right mental number line (Restle, 1970) with the spatial response layout. The aim of Experiment 2 was to evaluate if the findings of Experiment 1 extend to a non-spatial semantic (i.e., parity) classification task, using a design in which even for repetitions of the relevant attribute (i.e., parity) the irrelevant attribute (i.e., digit smaller vs. larger than 5) could be repeated without identical stimulus repetitions (e.g., a 7 followed by a 9 ). 


\section{Method}

\section{Participants}

Thirty-six University of Potsdam students, aged 18-31, with normal or corrected-to-normal vision participated in one session of approximately one hour. They either received a payment of 8 Euro or course credit for their participation.

\section{Stimuli and apparatus}

The stimuli were the digits (Verdana font, height $1.1 \mathrm{deg}$ ) 1-9 (excluding 5) presented at fixation; all other technical aspects were identical to Experiment 1.

\section{Procedure}

Twelve participants were randomly assigned to each group defined by the probability $\pi(=0.25,0.50,0.75)$ that the irrelevant numerical magnitude was repeated from one trial to the next. Following one practice block, in each of twelve blocks (comprising three warm-up trials, followed by 64 regular trials) each of the eight digits was shown eight times. Each of the four parity $\times$ magnitude combinations occurred 16 times, so that odd and even digits, and small and large digits occurred 32 times. In congruent trials either a small $(<5)$ digit required a left-hand response or a large $(>5)$ digit required a right-hand response; the other digitresponse combinations are incongruent. Parity (and thus the response) was repeated/alternated 32 times, the same was true of congruency status. For the $\pi=0.25(0.50,0.75)$ group, numerical magnitude ( $<5$ vs. $>5$ ) was repeated in $16(32,48)$ of the 64 trials in each block. Sequences were generated such that magnitude repetitions were independent of both parity and parity repetitions, and also of congruency status and congruency status repetitions. There were no trials in which the same digit as in the previous trial was presented.

The task was to indicate, as quickly as possible, the parity of the digit by a button press. Six participants in each group worked under each of both parity-to-response mappings (this variable had no main or interaction effect, and is ignored in the following). Each trial started with the presentation of a central fixation cross which after a delay of 500 ms was replaced with a response-terminated digit; the next trial then started after $1000 \mathrm{~ms}$.

All effects were tested at a significance level of $\alpha<$ .05 . For a trial to enter into the analysis of mean RT the response had to be correct, and be preceded by a correct response (exclusion rate of $8.8 \%$ ); also, RTs outside the range of $150 \mathrm{~ms}$ to $900 \mathrm{~ms}$ were excluded (a rate of $1.7 \%)$.

\section{Results}

\section{Mean RT}

We analyzed mean RT by a $3 \times 2 \times 2 \times 2$ mixed-model ANOVA in which the repetition probability of the irrelevant numerical magnitude was a between-subjects factor (3: $0.25,0.50,0.75$ ); three binary within-subject factors were defined by whether or not in the trial considered the irrelevant attribute (magnitude: digit $<5$ vs. digit $>5$ ) was repeated (2), the relevant attribute (parity) was repeated (2), and whether the trial was congruent or incongruent (2).

As shown in Fig. 3, response (i.e., parity) repetitions $(474 \mathrm{~ms})$ were faster than alternations $(484 \mathrm{~ms} ; \mathrm{F}(1,33)=$ $6.17, \mathrm{MSE}=6845, \eta^{2}=0.16, \mathrm{p}<.018$; right panel), and congruent (472 ms) trials led to shorter RTs than incongruent $(486 \mathrm{~ms})$ trials $($ SNARC effect; $\mathrm{F}(1,33)=13.90$, MSE $=905, \eta^{2}=0.30, p<.001 ;$ Fig. 4). In contrast to Experiment 1, repeating (477 ms) vs. alternating (482 ms) the irrelevant attribute (magnitude) also had a small but reliable main effect, $\mathrm{F}(1,33)=20.24, \mathrm{MSE}=88, \eta^{2}=0.38$, $\mathrm{p}$ $<.001$ (Fig. 3, left panel).

Critically, the interaction of the local magnitude repetition probability $\pi$ with actually repeating vs. alternating magnitude was significant $(\mathrm{F}(2,33)=7.34, \mathrm{MSE}=88$, $\left.\eta^{2}=0.31, \mathrm{p}<.002\right)$. Due to the main effect of magnitude repetition, the exact nature of this interaction takes a slightly different form than in Experiment 1, as shown in the left panel of Fig. 3. For the control group $(\pi=0.50)$, repeating numerical magnitude decreased mean RT by $5 \mathrm{~ms}$, relative to alternating it, a small but systematic magnitude repetition baseline effect $\left(t(11)=2.26, \eta^{2}=0.32, \mathrm{p}<0.045\right)$. For the $\pi=0.75$ group, this effect of repeating irrelevant magnitude is further increased by $5 \mathrm{~ms}$ to $10 \mathrm{~ms}(t(11)=4.76$, $\left.\eta^{2}=0.70, \mathrm{p}<0.01\right)$, and for $\pi=0.25$ it is decreased by the same amount, so that for this group repetitions and alternations of magnitude produced exactly the same mean RT $\left(t(11)=0.05, \eta^{2}=0.00, \mathrm{p}<0.96\right)$. The group factor defined by the three levels of $\pi$ neither modified the response repetition effect (Fig. 3, right panel; $F(2,33)=$ $\left.0.04, \mathrm{MSE}=1110, \eta^{2}=0.00, \mathrm{p}<.958\right)$ nor the SNARC effect Fig. 4; $\mathrm{F}(2,33)=1.83$, MSE $=905, \eta^{2}=0.10, \mathrm{p}<$ .176). The only other significant interaction was that when magnitude is repeated, parity (and thus response) repetitions are $15 \mathrm{~ms}$ faster; this response repetition benefit reduces to $5 \mathrm{~ms}$ when the numerical magnitude alternates $(\mathrm{F}(1,33)=$ 16.28, $\left.\mathrm{MSE}=130, \eta^{2}=0.33, \mathrm{p}<.001\right)$.

\section{Error rates}

The overall error rate (in\%) was 4.6. Fewer errors were made when magnitude was repeated (4.3) vs. alternated 


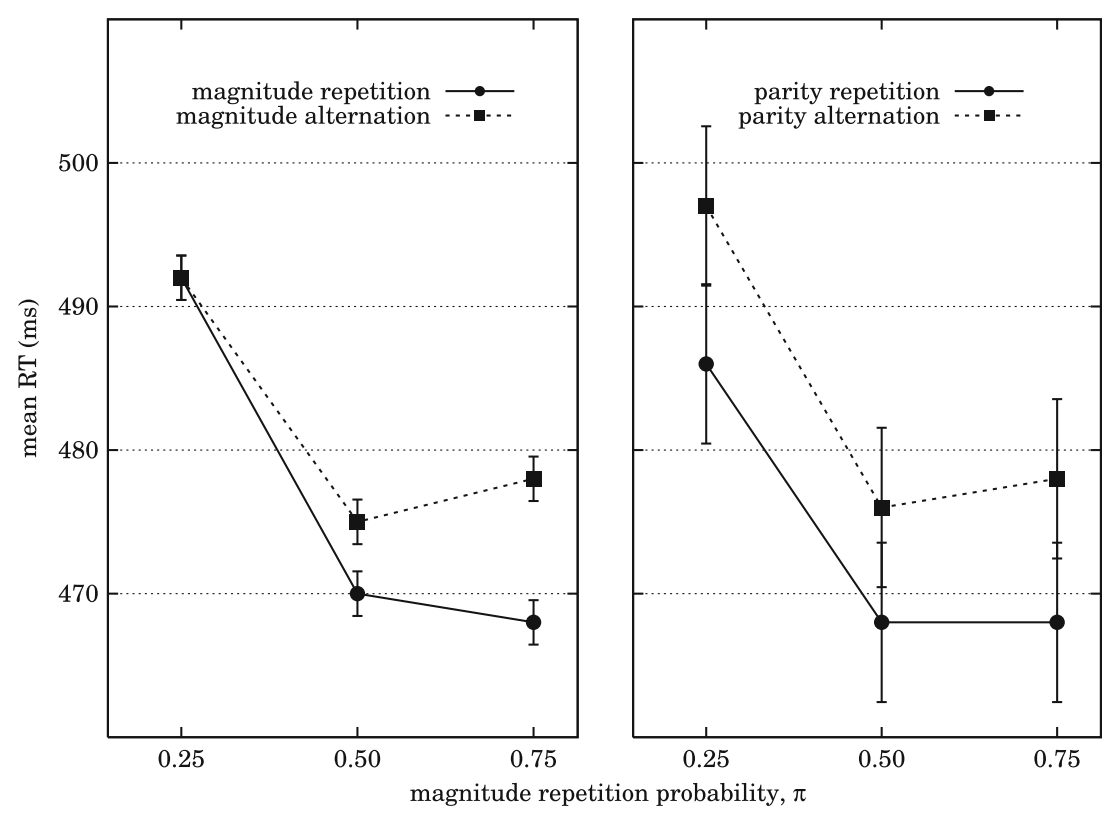

Fig. 3 Both panels: mean RT (ordinate in ms) for three groups with local probability of magnitude repetition equal to $\pi=0.25,0.50,0.75$ (abscissa). Left: mean RT for magnitude repetition (dots, solid lines) vs. magnitude alternation (squares, dotted lines) trials. Right: mean RT for parity (thus, response) repetition (dots, solid lines) vs. parity alternation (squares, dotted lines) trials. Error bars show \pm 1 s.e.m. (Loftus \& Massey, 1994)

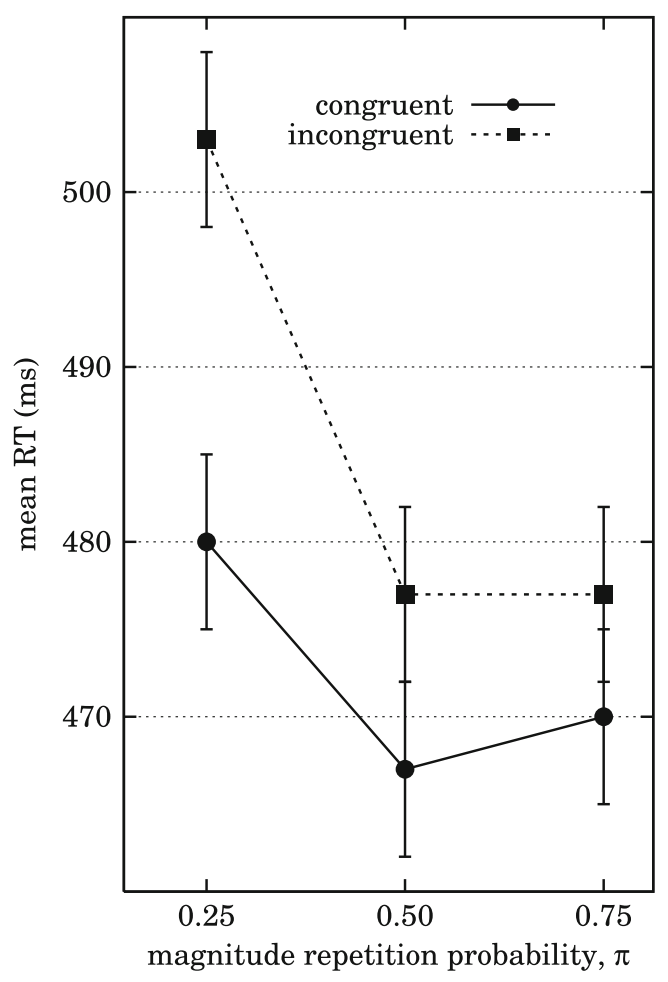

Fig. 4 Mean RT (ordinate in ms) for three groups with local probability of location repetition equal to $\pi=0.25,0.50,0.75$ (abscissa). Dots and solid lines indicate congruent, squares and dotted lines incongruent trials. Error bars show \pm 1 s.e.m. (Loftus \& Massey, 1994) $\left(5.0 ; \mathrm{F}(1,33)=4.90, \mathrm{MSE}=1.37, \eta^{2}=0.13, \mathrm{p}<.034\right)$, when parity was repeated (4.0) vs. alternated $(5.2 ; \mathrm{F}(1,33)$ $\left.=5.21, \mathrm{MSE}=18.59, \eta^{2}=0.14, \mathrm{p}<.029\right)$, and in congruent (3.5) vs. incongruent (5.7) trials $(\mathrm{F}(1,33)=11.31$, MSE $\left.=30.84, \eta^{2}=0.48, p<.002\right)$. The only significant interaction shows that fewer errors were made when magnitude and parity were both repeated or both alternated (4.2), compared to repeating one and alternating the other attribute $\left(5.1 ; \mathrm{F}(1,33)=9.91, \mathrm{MSE}=5.13, \eta^{2}=0.23, \mathrm{p}\right.$ $<.003)$. Given that none of the main or interaction effects involving the irrelevant repetition probability $\pi$ was significant we conclude that the RT findings regarding $\pi$ cannot be attributed to speed-accuracy trade-offs.

\section{Discussion}

The results of Experiment 2 confirm for a semantic classification task that variations of the local probability $\pi$ of repeating a response-irrelevant attribute (numerical magnitude) can have systematic and reliable effects on performance in a standard non-spatial conflict task. A notable difference to the Simon task (Experiment 1) is that in Experiment 2 repeating the irrelevant attribute per se reduced both RT and error rate. The combined pattern of both Experiments regarding this main effect thus closely parallels the mixed previous findings, reviewed in the Introduction, with no irrelevant location repetition main effects in visual Simon 
tasks (cf., Notebaert at al., 2001) but irrelevant flanker repetition main effects in letter classification tasks (cf., Kornblum \& Stevens, 2002).

The main effect of magnitude repetition indicates that across all levels of $\pi$ repeating similar numerical magnitudes from one trial to the next provides a genuine benefit which suggests one potential interpretation of the funnel interaction (rather than cross-over, as in Experiment 1, left panel of Fig. 1) of magnitude repetition probability $\pi$ with actually repeating vs. alternating magnitude. According to this interpretation, this interaction represents the additive combination of two separate components. One component reflects a genuine magnitude repetition effect that is independent of expectancies; it therefore arises even for the $\pi=0.50$ control group for which the stimulus sequence is completely unpredictable, admitting no expectancies above chance level. In contrast, the second component reflects expectancies based on learned trial contingencies, as were observed in Experiment 1. Specifically, participants in the $\pi=0.75$ group tend to expect magnitude repetitions: this leads to a benefit, on top of the genuine component, if magnitude is in fact repeated but to a cost if magnitude alternates. Participants in the $\pi=0.25$ group tend to expect magnitude alternations, with an ensuing benefit if in fact magnitude alternates; however, if contrary to those expectancies magnitude is repeated the cost of the expectancy mismatch is reckoned up against the benefit from the genuine component.

\section{General discussion}

An extensive body of research has established that repeating irrelevant but potentially conflicting stimulus attributes across trials can have reliable effects, under a wide range of well-understood boundary conditions (cf., Kornblum \& Stevens, 2002; Puccioni \& Vallesi 2012; Zeischka et al., 2010). To this research on sequential effects in filtering paradigms the present study adds as a new form of manipulation the local trial-to-trial transition probability $\pi$ of repeating a stimulus attribute that is logically and statistically irrelevant (i.e., unpredictive of the response required) yet potentially conflicting.

Local repetition probability (Bertelson, 1961; for reviews, see Jones et al., 2015; Soetens, 1998) has been extensively investigated before in connection with responserelevant attributes, but not in the context of response-irrelevant attributes in filtering paradigms. Because irrelevant local repetition probability was manipulated, events in the current trial provide a probabilistic basis to form expectancies about, for example, the location (Experiment 1) or the magnitude (Experiment 2) of the forthcoming stimulus. Our results show that these expectancies derived from learned design contingencies can influence how fast (Experiments 1 and 2) and accurate (Experiment 1) responses in the forthcoming trial are. Specifically, relative to the $\pi=0.50$ control groups working with unpredictive stimulus sequences, in both experiments participants in the $\pi=0.75$ groups responded faster when the irrelevant attribute was in fact repeated, and a complementary pattern was observed for groups with $\pi=0.25$. On the other hand, neither the reliable RT benefit associated with repeating the relevant attribute (and thus the response) nor the RT congruency main effects observed in both experiments were differentially modified by variations of $\pi$. Note that our results cannot be accounted for by some form of statistical association between the relevant and the irrelevant attribute, nor by an association of events in the present trial with the response or the congruency-status of the previous trial.

For the Simon task (Experiment 1), a natural interpretation is that the irrelevant attribute of the current trial acts much as a (mostly) valid centrally presented spatial cue for the next trial. Probabilistic knowledge about the location at which a critical stimulus will appear has well-documented facilitatory effects in valid trials, and detrimental effects in invalid trials (e.g., Foley \& Schwarz, 1998; Girardi, Antonucci, \& Nico, 2013, 2015; Gould, Wolfgang, \& Smith, 2007). This view is consistent with previous findings that location precues in Simon tasks produce the usual cost/benefit pattern but do not differentially influence the magnitude of the Simon effect (e.g., Lu \& Proctor, 1995; Wühr \& Kunde, 2008). Our interpretation also fits in, for example, with the attention-shift account of spatial correspondence effects (for recent review, see Duthoo et al., 2014; Puccioni \& Vallesi, 2012; Zeischka et al., 2010) according to which shifts of spatial attention contribute to the Simon effect as a key factor.

On this interpretation, why should irrelevant numerical magnitude in Experiment 2 also lead to corresponding effects of irrelevant repetition probability? According to a standard interpretation of number-related congruency effects one format in which we represent numbers is topologically akin to the spatial stimulus layout in a Simon task, the fundamental difference being, of course, that location in the parity task corresponds to the internal location of a number along an ordered quasi-spatial representation (the "mental number line"; Restle, 1970). In this interpretation, expectancy effects regarding sub-intervals of the mental number line may facilitate the identification of the to-bepresented number (e.g., Schwarz \& Ischebeck, 2000; Tan \& Dixon, 2011), and thus of its parity, not unlike a valid spatial location cue in a Simon task. Considerable support for this view is, for example, provided by the work of Nieder (e.g., 2005, 2011) who explored basic neuronal mechanisms of numerical magnitude coding. Recording from cells in the intraparietal sulcus and the prefrontal cortex of rhesus 
monkeys, he identified a numerical distance-dependent gradient of neuronal activation, yielding well-defined neuronal numerosity-tuning functions for single cells. If this distancedependent mechanism is transiently facilitated by residual activation from the previous trial, then repeating digits of similar magnitude in subsequent trials might facilitate an initial orientation response along the mental number line.

More generally, expectancy effects as induced by local repetition probability offer a promising approach to further explore how stimulus attributes are processed which by themselves are uniformative about the required response but (in typical filtering paradigms) do induce reliable response repetition and/or congruency effects. Two aspects of this approach seem especially relevant in the present context. The first is that changes of the global probability of stimulus attributes imply changes of its local first-order transition probabilities, too, whereas the converse is not true (cf., Kornblum, 1968; Miller, 1998). For example, if in a Simon task as used in Experiment 1 in any trial the stimulus is independently presented in the left location with probability $0.80(0.50)$, then 100 trials will on average contain 68 (50) first-order location repetitions. This confound makes it difficult to attribute the effects of varying the global probability of irrelevant attributes either to cumulative overall learning effects (due simply to more frequent presentations of one location; cf., Kabata \& Matsumoto, 2012; Miller, $1988,1998)$ or to more local, expectancy-related sequential effects. In contrast, the local repetition probability $\pi$ of irrelevant stimulus attributes can be manipulated without altering the global (overall) probability of that attribute. Second, it is difficult to attribute the local repetition effects of twovalued relevant attributes in two-choice paradigms (such as color in Experiment 1, or parity in Experiment 2) to chronometrically early (stimulus-related) or late (response-related) processing stages, as repetitions of the relevant attribute and of the associated response are perfectly confounded in such designs. In contrast, the local repetition effects of irrelevant attributes obtained in the present study are independent both of the relevant stimulus attribute (and thus the response) and of congruity status, and also of whether or not these attributes have been repeated from the previous trial or not. Conflict paradigms are by definition based on manipulating the relation of relevant and irrelevant stimulus attributes; given that the variation of the local repetition probability of irrelevant attributes avoids the confounds mentioned above, the general design behind the present experiments allows for cleaner conclusions about how expectancy effects modulate the adaptation to inconsistent or contradictory information.

In conclusion, the present experiments demonstrate systematic and reliable effects due to variations of the local trial-to-trial repetition probability of response-irrelevant attributes (location, numerical magnitude) in a spatial (Simon) and in a semantic (SNARC) filtering task. These effects influence performance in ways comparable to central probabilistic location (Experiment 1) or magnitude (Experiment 2) cues: they exhibit the cost-benefit pattern typically associated with such cues, but do not in turn differentially modify other main (response repetition and congruency) effects. More generally, the design used in our experiments provides a novel approach to explore how trial-to-trial expectancies regarding response-irrelevant attributes influence the nature and limits of cognitive control in filtering tasks.

Author Note Wolf Schwarz, University of Potsdam, Faculty of Humanities, PO Box 6015 53, D - 14415 Potsdam, Germany. Email: wschwarz@uni-potsdam.de (to whom all correspondence should be addressed).

Dennis Reike, University of Potsdam, Faculty of Humanities, PO Box 6015 53, D - 14415 Potsdam, Germany. Email: reike@ unipotsdam.de

Acknowledgments We would like to thank Prof. Jeff Miller, University of Otago, New Zealand, for helpful suggestions and discussions regarding the present work.

The present research was funded by the Deutsche Forschungsgemeinschaft (DFG; grant no. Schw 611/3-1).

\section{References}

Bertelson, P. (1961). Sequential redundancy and speed in a serial two-choice responding task. Quarterly Journal of Experimental Psychology, 13, 90-102.

Blais, C., Harris, M.B.H., Guerrero, J.V., \& Bunge, S.A. (2012). Rethinking the role of automaticity in cognitive control. The Quarterly Journal of Experimental Psychology, 65, 268276.

Botvinick, M.M., Braver, T.S., Barch, D.M., Carter, C.S., \& Cohen, J.D. (2001). Conflict monitoring and cognitive control. Quarterly Journal of Experimental Psychology, 108, 624-652.

Bugg, J.M., Diede, N.T., Cohen-Shikora, E.R., \& Selmeczy, D. (2015). Expectations and experience: dissociable bases for cognitive control? Journal of Experimental Psychology: Learning, Memory, and Cognition, 41, 1349-1373.

Dehaene, S. (1996). The organization of brain activations in number comparison: event-related potentials and the additive-factors method. Journal of Cognitive Neuroscience, 8, 47-68.

Dehaene, S. (2011). The number sense: how the mind creates mathematics, revised and updated edition. New York: Oxford University Press.

Dehaene, S., Bossini, S., \& Giraux, P. (1993). The mental representation of parity and numerical magnitude. Journal of Experimental Psychology: General, 122, 371-396.

Duthoo, W., Abrahamse, E.L., Braem, S., Boehler, C.N., \& Notebaert, W. (2014). The heterogeneous world of congruency sequence effects: an update. Frontiers in Psychology, 5.

Eriksen, B.A., \& Eriksen, C.W. (1974). Effects of noise letters upon the identification of a target letter in a nonsearch task. Perception \& Psychophysics, 16, 143-149.

Fias, W., \& Fischer, M.H. (2005). Spatial representation of numbers. In J.I.D. Campbell (Ed.), Handbook of mathematical cognition, (pp. 43-54). New York: Psychology Press. 
Foley, J., \& Schwarz, W. (1998). Spatial attention: effect of position uncertainty and number of distractor patterns on the threshold vs. contrast $(\mathrm{TvC})$ function for contrast discrimination. Journal of the Optical Society of America, A, 15, 1036-1047.

Frings, C., Rothermund, K., \& Wentura, D. (2007). Distractor repetitions retrieve previous responses to targets. The Quarterly Journal of Experimental Psychology, 60, 1367-1377.

Girardi, G., Antonucci, G., \& Nico, D. (2013). Cueing spatial attention through timing and probability. Cortex, 49, 211-221.

Girardi, G., Antonucci, G., \& Nico, D. (2015). Timing the events of directional cueing. Psychological Research, 79, 1009-1021.

Gould, I.C., Wolfgang, B.J., \& Smith, P.L. (2007). Spatial uncertainty explains exogenous and endogenous attentional cuing effects in visual signal detection. Journal of Vision, 7.

Gratton, G., Coles, M.G.H., \& Donchin, E. (1992). Optimizing the use of information: strategic control of activation of responses. Journal of Experimental Psychology: General, 121, 480-506.

Hommel, B. (2011). The Simon effect as tool and heuristic. Acta Psychologica, 136, 189-202.

Jentzsch, I., \& Leuthold, H. (2005). Response conflict determines sequential effects in serial response time tasks with short responsestimulus intervals. Journal of Experimental Psychology: Human Perception and Performance, 31, 731-748.

Jiménez, L., \& Méndez, A. (2013). It is not what you expect: dissociating conflict adaptation from expectancies in a Stroop task. Journal of Experimental Psychology: Human Perception and Performance, 39, 271-284.

Jiménez, L., \& Méndez, A. (2014). Even with time, conflict adaptation is not made of expectancies. Frontiers in Psychology, 5.

Jones, M., Curran, T., Mozer, M.C., \& Wilder, M.H. (2015). Sequential effects in response time reveal learning mechanisms and event representations. Psychological Review, 120, 628-666.

Jongen, E.M.M., \& Smulders, F.T.Y. (2007). Sequence effects in a spatial cueing task: endogenous orienting is sensitive to orienting in the preceding trial. Psychological Research, 71, 516523.

Kabata, T., \& Matsumoto, E. (2012). Cueing effects of target location probability and repetition. Vision Research, 73, 23-29.

Kahneman, D., \& Treisman, A. (1984). Changing views of attention and automaticity. In R. Parasuraman, \& D.R. Davies (Eds.), Varieties of attention. New York: Academic Press.

Kleinsorge, T. (1999). Response repetition benefits and costs. Acta Psychologica, 103, 295-310.

Kornblum, S. (1968). Serial-choice reaction time: inadequacies of the information hypothesis. Science, 159, 432-434.

Kornblum, S., \& Stevens, G. (2002). Sequential effects in dimensional overlap: findings and issues. In W. Prinz, \& B. Hommel (Eds.), Common mechanisms in perception and action, attention and performance, Vol. 19. London: Oxford University Press.

Leuthold, H., \& Sommer, W. (1993). Stimulus presentation rate dissociates sequential effects in event-related potentials and reaction times. Psychophysiology, 30, 510-517.

Loftus, G.R., \& Masson, M.E.J. (1994). Using confidence intervals in within-subject designs. Psychonomic Bulletin \& Review, 1, 476490.

Lu, C., \& Proctor, R.W. (1995). The influence of irrelevant location information on performance: a review of the Simon and spatial Stroop effects. Psychonomic Bulletin \& Review, 2, 174-207.

Marcel, T., \& Forrin, B. (1974). Naming latency and the repetition of stimulus categories. Journal of Experimental Psychology, 103, 450-460.

MacLeod, C.M. (1991). Half a century of research on the Stroop effect: an integrative review. Psychological Bulletin, 109, 163-203.

Mayr, U., Awh, E., \& Laurey, P. (2003). Conflict adaptation effects in the absence of executive control. Nature Neuroscience, 6, 450 452 .
Meuter, R.F.I., \& Allport, A. (1999). Bilingual language switching in naming: asymmetrical costs of language selection. Journal of Memory and Language, 40, 25-40.

Miller, J. (1988). Components of the location probability effect in visual search tasks. Journal of Experimental Psychology: Human Perception and Performance, 14, 453-471.

Miller, J. (1991). The flanker compatibility effect as a function of visual angle, attentional focus, visual transients, and perceptual load: a search for boundary conditions. Perception \& Psychphysics, 49, 270-288.

Miller, J. (1998). Effects of stimulus-response probability on choice reaction time: evidence from the lateralized readiness potential. Journal of Experimental Psychology: Human Perception and Performance, 24, 1521-1534.

Nieder, A. (2005). Counting on neurons: the neurobiology of numerical competence. Nature Reviews Neuroscience, 6, 177-190.

Nieder, A. (2011). The neural code for number. In S. Dehaene, \& E.M. Brannon (Eds.), Space, time and number in the brain. Searching for the foundations of mathematical thought, chap, 8, (pp. 103118). London: Academic Press.

Notebaert, W., \& Soetens, E. (2003). The influence of irrelevant stimulus changes on stimulus and response repetition effects. Acta Psychologica, 112, 143-156.

Notebaert, W., Soetens, E., \& Melis, A. (2001). Sequential analysis of a Simon task-evidence for an attention-shift account. Psychological Research, 65, 170-184.

Pashler, H. (1998). The psychology of attention. London: The MIT Press.

Pashler, H., \& Baylis, G. (1991). Procedural learning: intertrial repetition effects in speeded-choice tasks. Journal of Experimental Psychology: Learning, Memory, and Cognition, 17, 33-48.

Pfister, R., Schroeder, P.A., \& Kunde, W. (2013). SNARC struggles: instant control over spatial-numerical associations. Journal of Experimental Psychology: Learning, Memory, and Cognition, 39, 1953-1958.

Pratte, M.S., Rouder, J.N., Morey, R.D., \& Feng, C. (2010). Exploring the differences in distributional properties between Stroop and Simon effects using delta plots. Attention, Perception \& Psychophysics, 72, 2013-2025.

Puccioni, O., \& Vallesi, A. (2012). Sequential congruency effects: disentangling priming and conflict adaptation. Psychological Research, 76, 591-600.

Restle, F. (1970). Speed of adding and comparing numbers. Journal of Experimental Psychology, 83, 274-278.

Rothermund, K., Wentura, D., \& De Houwer, J. (2005). Retrieval of incidental stimulus-response associations as a source of negative priming. Journal of Experimental Psychology: Learning, Memory, and Cognition, 31, 482-495.

Schmidt, J.R. (2013). Questioning conflict adaptation: proportion congruent and Gratton effects reconsidered. Psychonomic Bulletin \& Review, 20, 615-630.

Schmidt, J.R., \& Besner, D. (2008). The Stroop effect: Why proportion congruent has nothing to do with congruency and everything to do with contingency. Journal of Experimental Psychology: Learning, Memory, and Cognition, 34, 514-523.

Schmidt, J.R., \& De Houwer, J. (2012a). Contingency learning with evaluative stimuli testing the generality of contingency learning in a performance paradigm. Experimental Psychology, 59, 175182.

Schmidt, J.R., \& De Houwer, J. (2012b). Does temporal contiguity moderate contingency learning in a speeded performance task? The Quarterly Journal of Experimental Psychology, 65, 408-425.

Schmidt, J.R., Crump, M.J.C., Cheesman, J., \& Besner, D. (2007). Contingency learning without awareness: evidence for implicit control. Consciousness and Cognition, 16, 421-435. 
Schmidt, J.R., De Houwer, J., \& Besner, D. (2010). Contingency learning and unlearning in the blink of an eye: a resource dependent process. Consciousness and Cognition, 19, 235-250.

Schwarz, W., \& Ischebeck, A. (2000). Sequential effects in number comparison. Journal of Experimental Psychology: Human Perception and Performance, 26, 1606-1621.

Schwarz, W., \& Ischebeck, A. (2003). On the relative speed account of number-size interference effects in comparative judgments of numerals. Journal of Experimental Psychology: Human Perception and Performance, 29, 507-522.

Schwarz, W., \& Keus, I.M. (2004). Moving the eyes along the mental number line: comparing SNARC effects with saccadic and manual responses. Perception \& Psychophysics, 66, 651-664.

Schwarz, W., \& Müller, D. (2006). Spatial associations in numberrelated tasks: a comparison of manual and pedal responses. Experimental Psychology, 53, 4-15.

Simon, J.R., \& Berbaum, K. (1990). Effect of conflicting cues on information processing: the "Stroop effect" vs. the "Simon effect". Acta Psychologica, 73, 159-170.
Stroop, J.R. (1935). Studies of interference in serial verbal reactions. Journal of Experimental Psychology, 18, 643-662.

Soetens, E. (1998). Localizing sequential effects in serial choice reaction time with the information reduction procedure. Journal of Experimental Psychology: Human Perception and Performance, 24, 547-568.

Tan, S., \& Dixon, P. (2011). Repetition and the SNARC effect with one- and two-digit numbers. Canadian Journal of Experimental Psychology, 65, 84-97.

Wühr, P., \& Ansorge, U. (2005). Exploring trial-by-trial modulations of the Simon effect. The Quarterly Journal of Experimental Psychology Section A: Human Experimental Psychology, 58, 705-731.

Wühr, P., \& Kunde, W. (2008). Precueing spatial S-R correspondence: Is there regulation of expected response conflict? Journal of Experimental Psychology: Human Perception and Performance, $34,872-883$.

Zeischka, P., Deroost, N., Maetens, K., \& Soetens, E. (2010). Reduced congruency effects only for repeated spatial irrelevant information. European Journal of Cognitive Psychology, 22, 1137-1167. 